\title{
A NOTE ON COHOMOLOGICAL DIMENSION OF APPROXIMATE MOVABLE SPACES
}

\author{
TADASHI WATANABE
}

(Communicated by James E. West)

\begin{abstract}
We show that any approximate movable compact metric space $X$ satisfies the equality $\operatorname{dim} X=\operatorname{dim}_{\mathrm{Z}} X$ without finite dimensional condition. Thus there is no approximate movable compact metric space $X$ with $\operatorname{dim} X=$ $\infty$ and $\operatorname{dim}_{\mathrm{Z}} X<\infty$. Since ANRs and some generalized ANRs are approximate movable, they satisfy the above equality.
\end{abstract}

All spaces are compact metric and all polyhedra are finite. Let $X$ be a space. By $\operatorname{dim} X$ and $\operatorname{dim}_{\mathbb{Z}} X$ we denote covering dimension and integral cohomological dimension of $X$, respectively. It is well known (the fundamental cohomological dimension theorem) that if $\operatorname{dim} X$ is finite, then $\operatorname{dim} X=\operatorname{dim}_{\mathbb{Z}} X$ (see P. S. Aleksandrov [1]). Recently, A. N. Dranishnikov [5] constructed a space $X$ with $\operatorname{dim} X=\infty$ and $\operatorname{dim}_{\mathbb{Z}} X=3$. So his example means that the equality $\operatorname{dim} X=\operatorname{dim}_{\mathbb{Z}} X$ does not hold without finite dimensional condition. In this note we investigate this equality for some nice spaces:

Theorem 1. If $X$ is approximate movable, then $\operatorname{dim} X=\operatorname{dim}_{\mathbb{Z}} X$ holds.

Corollary 2. There does not exist an approximate movable space $X$ with $\operatorname{dim} X$ $=\infty$ and $\operatorname{dim}_{\mathbb{Z}} X<\infty$.

In [9] the author introduced an approximate shape theory and approximate movability which is an approximate invariant property.

Let $X$ be a space, and let $\mathscr{X}=\left\{P_{i}, f_{i j}, \mathbb{N}\right\}$ be an inverse sequence of polyhedra $P_{i}$ and maps $f_{j i}: X_{j} \rightarrow X_{i}, i<j$, such that $X$ is an inverse limit of $\mathscr{X}$. Lemma (1.6) of $[9, \mathrm{II}]$ means the following:

Lemma 3. $X$ is approximate movable if and only if for each integer $k$ and each $\varepsilon>0$ there is an integer $j>k$ with the following property: For each integer $i \geq k$ there is a map $r_{i}: X_{j} \rightarrow X_{i}$ such that $f_{i k} r_{i}$ and $f_{j k}$ are $\varepsilon$-near.

For our proof we need some characterizations of dimension and cohomological dimension. For any integer $n$ and any triangulation $K, K^{(n)}$ denotes the $n$-th skeleton of $K$ and $|K|$ denotes the realization of $K$. Lemmas 4 and 5 are Theorem 4.1 and Theorem 5.1 of [8].

Received by the editors November 15, 1993.

1991 Mathematics Subject Classification. Primary 54C55, 54C56, 54F45.

Key words and phrases. Covering dimension, cohomological dimension, ANR, generalized ANR, approximate movability. 
Lemma 4. $X$ has $\operatorname{dim} X \leq n$ if and only if for each integer $k$ and each $\varepsilon>0$ there exist an integer $j>k$, a triangulation $L_{k}$ of $P_{k}$, and a map $g_{j k}: P_{j} \rightarrow$ $\left|L_{k}^{(n)}\right|$ which is $\varepsilon$-close to $f_{j k}$.

Lemma 5 (R. D. Edwards). $X$ has $\operatorname{dim}_{\mathbb{Z}} X \leq n$ if and only if, given an integer $i \geq 1$, for each integer $k$ and each $\varepsilon>0$ there is a triangulation $L_{k}$ of $P_{k}$ and an integer $j>k$ such that for any triangulation $L_{j}$ of $P_{j}$ there is a map $g_{j k}:\left|L_{j}^{(n+i)}\right| \rightarrow\left|L_{k}^{(n)}\right|$ which is $\varepsilon$-close to the restriction of $f_{j k}$.

Proof of Theorem 1. First, we show the inequality $\operatorname{dim} X \leq \operatorname{dim}_{\mathbb{Z}} X$. If $\operatorname{dim}_{\mathbb{Z}} X$ $=\infty$, there is nothing to prove, so we consider the case $\operatorname{dim}_{\mathbb{Z}} X \leq n<\infty$ for some integer $n$. Take any integer $k$ and any $\varepsilon>0$. Put $\delta=\varepsilon / 3$. Since $X$ is approximate movable, by Lemma 3 there is an integer $j \geq k$ satisfying

(1) for each $i \geq k$ there is a map $r_{i}: P_{j} \rightarrow P_{i}$ such that $f_{i k} r_{i}$ and $f_{j k}$ are $\delta$-near.

Since $P_{j}$ is a finite polyhedron, take a triangulation $L_{j}$ of $P_{j}$ and let $s=$ $\operatorname{dim} L_{j}<\infty$. Since $\operatorname{dim}_{\mathbb{Z}} X \leq n<\infty$, by Lemma 5 there exist a triangulation $L_{k}$ of $P_{k}$ and an integer $i>k$ such that

(2) for any triangulation $L_{i}$ of $P_{i}$ there is a map $g_{i k}:\left|L_{i}^{(n+s)}\right| \rightarrow\left|L_{k}^{(n)}\right|$ which is $\delta$-close to the restriction of $f_{i k}$.

Since $f_{i k}: X_{i} \rightarrow X_{k}$ is uniform, there is an $\eta>0$ such that if points $x$ and $x^{\prime}$ in $X_{i}$ are $\eta$-near, then $f_{i k}(x)$ and $f_{i k}\left(x^{\prime}\right)$ are $\delta$-near. Take a triangulation $L_{i}$ of $P_{i}$ such that any simplex of $L_{i}$ has a diameter $<\eta / 2$. By the simplicial approximation theorem there are a subdivision $L_{j}^{\prime}$ of $L_{j}$ and a simplicial map $\varphi: L_{j}^{\prime} \rightarrow L_{i}$ which approximates $r_{i}$, i.e., its realization $|\varphi|$ and $r_{i}$ are $\eta$-near. By the choice of $\eta, f_{i k}|\varphi|$ and $f_{i k} r_{i}$ are $\delta$-near. Since $\varphi$ is simplicial and $s=\operatorname{dim} L_{j}=\operatorname{dim} L_{j}^{\prime}, \varphi$ induces a map $h=|\varphi|: P_{j}=\left|L_{j}^{\prime}\right|=\left|L_{j}^{\prime(s)}\right| \rightarrow\left|L_{i}^{(s)}\right| \subset$ $\left|L_{i}^{(n+s)}\right|$. Thus

(3) $f_{i k} h$ and $f_{i k} r_{i}$ are $\delta$-near.

Since $h: P_{j} \rightarrow\left|L_{i}^{(n+s)}\right|$, by (2)

(4) $g_{i k} h$ and $f_{i k} h$ are $\delta$-near.

By (1), (3) and (4), $f_{j k}$ and $g_{i k} h: P_{j} \rightarrow\left|L_{k}^{(n)}\right|$ are $\varepsilon$-near. Thus $j$ and the map $g_{i k} h$ satisfies the condition in Lemma 4 for $k$ and $\varepsilon$. Then $\operatorname{dim} X \leq n$. This means the inequality $\operatorname{dim} X \leq \operatorname{dim}_{\mathbb{Z}} X$.

Next, we show the inequality $\operatorname{dim}_{\mathbb{Z}} X \leq \operatorname{dim} X$. If $\operatorname{dim} X=\infty$, there is nothing to prove, so we consider the case $\operatorname{dim} X \leq n<\infty$ for some integer $n$. It is easy to show $\operatorname{dim}_{\mathbb{Z}} X \leq n$ by Lemmas 4 and 5 . This means the inequality $\operatorname{dim}_{\mathbb{Z}} X \leq \operatorname{dim} X$. Therefore, we have the required equality.

Corollary 2 follows from Theorem 1 and also means that Dranishnikov's example is not approximate movable.

Borsuk [2] introduced an absolute neighborhood retract, in notation ANR. There are many generalizations of ANR. Noguchi [7] introduced an absolute neighborhood retract in the sense of Noguchi, in notation $A_{A N R_{N}}$. Clapp [4] introduced an absolute neighborhood retract in the sense of Clapp, in notation $\mathrm{AANR}_{\mathrm{C}}$. Borsuk [3] introduced a nearly extendable set, in notation NE-set. Mardešić [6] introduced an approximate polyhedron, in notation AP. In [9, II, III] we gave their descriptions in approximate shape theory. By (II.4.7), (II.4.6), (II.2.18), (II.2.3), (II.5.10), (II.5.11) and (III.1.2) of [9], these ANR, 
$\mathrm{AANR}_{\mathrm{N}}, \mathrm{AANR}_{\mathrm{C}}, \mathrm{NE}$-set and AP are approximate movable (see the table of $[9$, II, p. 337]). Thus we have

Corollary 6. If $X$ is $A N R, A N R_{N}, A N R_{C}, N E$-set or $A P$, then $\operatorname{dim} X=\operatorname{dim}_{\mathbb{Z}} X$ holds.

\section{REFERENCES}

1. P. S. Aleksandrov, Dimensiontheorie, ein Betrag zur Geometrie der abgeschlossen Mengen, Math. Ann. 106 (1932), 161-238.

2. K. Borsuk, Theory of retracts, Monograf. Mat., vol. 44, Polish Scientific Publishers, Warszawa, 1967.

3. __ On a class of compacta, Houston J. Math. 1 (1975), 1-13.

4. M. H. Clapp, On a generalization of absolute neighborhood retracts, Fund. Math. 70 (1971), 117-130.

5. A. N. Dranishnikov, On a problem of P. S. Alexsandroff, Mat. Sb. 135 (177) (1988), no. 4, 551-557.

6. S. Mardešić, Approximate polyhedra, resolutions of maps and shape fibrations, Fund. Math. 114 (1981), 53-78.

7. H. Noguchi, A generalization of absolute neighborhood retracts, Kodai Math. Seminar Report 1 (1953), 20-22.

8. J. J. Walsh, Dimension, cohomological dimension, and cell-like mappings, Shape Theory and Geom. Top. Proc. (Dubrovnik, 1981), Lecture Notes in Math., vol. 870, Springer-Verlag, Berlin and New York, 1981, pp. 105-118.

9. T. Watanabe, Approximative shape I-IV, Tsukuba J. Math. 11 (1987), 17-59; 11 (1987), 303-339; 12 (1988), 1-41; 12 (1989), 273-319.

Department of Mathematics, Faculty of Education, University of Yamaguchi, YamAGUCHI CITY, 753, JAPAN

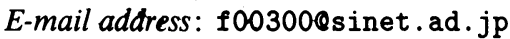

